OPEN ACCESS

Edited by: Soman Ninan Abraham,

Duke University, United States

Reviewed by:

Gregorio Gomez,

University of Houston,

United States

Carlos Muñoz-Garay,

Universidad Nacional Autónoma

de México, Mexico

*Correspondence:

Hydar Ali

alih@upenn.edu

Specialty section:

This article was submitted to

Microbial Immunology,

a section of the journal

Frontiers in Immunology

Received: 31 March 2021 Accepted: 08 June 2021

Published: 23 June 2021

Citation:

Amponnawarat $A$,

Chompunud Na Ayudhya C and Ali $H$ (2021) Murepavadin,

a Small Molecule Host Defense

Peptide Mimetic, Activates Mast Cells via MRGPRX2 and MrgprB2.

Front. Immunol. 12:689410. doi: 10.3389/fimmu.2021.689410

\section{Murepavadin, a Small Molecule Host Defense Peptide Mimetic, Activates Mast Cells via MRGPRX2 and MrgprB2}

\author{
Aetas Amponnawarat ${ }^{1,2}$, Chalatip Chompunud Na Ayudhya ${ }^{1,3}$ and Hydar Ali ${ }^{\text {* }}$ \\ ${ }^{1}$ Department of Basic and Translational Sciences, School of Dental Medicine, University of Pennsylvania, Philadelphia, \\ $P A$, United States, ${ }^{2}$ Department of Family and Community Dentistry, Faculty of Dentistry, Chiang Mai University, \\ Chiang Mai, Thailand, ${ }^{3}$ Department of Oral Diagnosis, Faculty of Dentistry, Naresuan University, Phitsanulok, Thailand
}

Pseudomonas aeruginosa is a frequent cause of hospital-acquired wound infection and is difficult to treat because it forms biofilms and displays antibiotic resistance. Previous studies in mice demonstrated that mast cells (MCs) not only contribute to $P$. aeruginosa eradication but also promote wound healing via an unknown mechanism. We recently reported that host defense peptides (HDPs) induce human MC degranulation via Masrelated G protein-coupled receptor-X2 (MRGPRX2). Small molecule HDP mimetics have distinct advantages over HDPs because they are inexpensive to synthesize and display high stability, bioavailability, and low toxicity. Murepavadin is a lipidated HDP mimetic, (also known as POL7080), which displays antibacterial activity against a broad panel of multi-drug-resistant $P$. aeruginosa. We found that murepavadin induces $\mathrm{Ca}^{2+}$ mobilization, degranulation, chemokine IL-8 and CCL3 production in a human MC line (LAD2 cells) endogenously expressing MRGPRX2. Murepavadin also caused degranulation in RBL-2H3 cells expressing MRGPRX2 but this response was significantly reduced in cells expressing missense variants within the receptor's ligand binding (G165E) or G protein coupling (V282M) domains. Compound 48/80 induced $\beta$ arrestin recruitment and promoted receptor internalization, which resulted in substantial decrease in the subsequent responsiveness to the MRGPRX2 agonist. By contrast, murepavadin did not cause $\beta$-arrestin-mediated MRGPRX2 regulation. Murepavadin induced degranulation in mouse peritoneal MCs via MrgprB2 (ortholog of human MRGPRX2) and caused increased vascular permeability in wild-type mice but not in $\mathrm{MrgprB2}^{-/-}$mice. The data presented herein demonstrate that murepavadin activates human MCs via MRGPRX2 and murine MCs via MrgprB2 and that MRGPRX2 is resistant to $\beta$-arrestin-mediated receptor regulation. Thus, besides its direct activity against $P$. aeruginosa, murepavadin may contribute to bacterial clearance and promote wound healing by harnessing MC's immunomodulatory property via the activation of MRGPRX2.

Keywords: murepavadin, mast cells, MrgprB2, MRGPRX2, host defense peptides, antimicrobial peptides 


\section{INTRODUCTION}

The emergence of multidrug-resistant bacterial infections poses a global public health threat that warrants urgent need for alternative therapeutic approaches (1). Host defense peptides (HDPs), previously known as antimicrobial peptides (AMPs), such as the cathelicidin LL-37 and human $\beta$-defensins are considered as promising antimicrobial agents (2-4). Recent evidence demonstrated that in addition to their direct antimicrobial activity, HDPs promote the recruitment and activation of various immune cells including mast cells (MCs), neutrophils, monocytes and lymphocytes (5-8). These HDPs also display angiogenic activity and contribute to wound healing $(8,9)$. However, many HDPs cause the lysis of erythrocytes and display cytotoxicity against a variety of cells $(10,11)$. In recent years, great strides have been made in optimizing HDPs to minimize their toxicity and to improve their stability, which can also modulate the immune system for therapeutic benefits $(12,13)$.

Mast cells (MCs) are multifunctional immune cells of hematopoietic origin that are found in vascularized tissues such as the oral mucosa, intestine, airway and the skin. MCs play an important role in host defense and promote wound healing (1416). In addition to high affinity IgE receptor (FceRI), a subtype of human $\mathrm{MCs}\left(\mathrm{MC}_{\mathrm{TC}}\right.$; contain both tryptase and chymase) expresses a G protein-coupled receptor (GPCR) known as Mas-related GPCR-X2 (MRGPRX2) $(17,18)$. This receptor is highly expressed in human skin MCs but is also present in lung and gut MCs but at lower levels $(17,19,20)$. Mouse connective tissue MCs (CTMC; skin, nasopharynx and peritoneal) express MrgprB2 (ortholog of human MRGPRX2) (16, 21). Both receptors are activated by human and mouse HDPs (22-24). Studies with human MCs expressing MRGPRX2 and MrgprB2 ${ }^{-1-}$ mice have strongly implicated these receptors in innate immunity and wound healing $(15,16)$. The major portal of entry for pathogen is the interface between host and external microenvironment such as the skin, nasopharynx, and peritoneum. MrgprB2-expressing CTMCs are found abundantly at these sites and contribute to host defense against bacterial infection through the release of MC-derived mediators and the subsequent recruitment of neutrophils (16). Furthermore, pharmacological activation of MrgprB2 at these sites results in decreased bacterial count and reduced disease severity in vivo (16).

Pseudomonas aeruginosa is a Gram-negative bacterium that often presents a therapeutic challenge due to its ability to form biofilms and to display antibiotic resistance $(25,26)$. Zimmerman et al. (27), utilized a topical $P$. aeruginosa infection model and demonstrated that MCs contribute to both bacterial elimination and promote wound healing. However, they found that culturing MCs infected with $P$. aeruginosa in vitro is insufficient to eliminate bacteria unless they are co-cultured with keratinocytes. MC mediators released in response to $P$. aeruginosa infection results in the secretion of HDPs such as mouse $\beta$-defensin-14 (Defb14, ortholog of human $\beta$-defensin-3) from keratinocytes (27). These findings suggest that MC-derived mediators confer protective immunity through the promotion of endogenous HDP secretion, which in turn, directly kill the bacteria and restrain the infection (27).
Protegrin-1 is a HDP that was originally purified from porcine leukocytes (28). We have recently shown that protegrin-1 activates human MCs via MRGPRX2 (29). However, small molecule HDP mimetics have a number of advantages over natural HDPs because of their superior stability, bioavailability, and reduced toxicity $(30,31)$. Moreover, several approaches have been used to increase hydrophobicity and membrane activity of HDP mimetics (32). Murepavadin is a lipidated protegrin-1 mimetic, (also known as POL7080), which specifically targets $P$. aeruginosa, including multidrug-resistant clinical isolates (33). Thus, it could be used for the treatment of antibiotic-resistant $P$. aeruginosa skin infection $(34,35)$. Given that HDPs including protegrin-1 activate human MCs via MRGPRX2 (22, 23, 29), raises the interesting possibility that potential therapeutic action of murepavadin for $P$. aeruginosa skin infection likely reflects both MRGPRX2-mediated MC activation and its direct antimicrobial activity. However, the possibility that murepavadin activates MCs has not been tested.

Besides G proteins, most GPCR agonists activate another signaling pathway that requires the recruitment of adapter proteins known as $\beta$-arrestins. This $\beta$-arrestin-mediated pathway was first identified for its role in receptor desensitization (uncoupling of receptor/G protein interaction) and internalization (36). Agonists that prefer to activate $G$ proteins over $\beta$-arrestins are known as $G$ protein-biased agonists, whereas agonists that selectively activate $\beta$-arrestins are known as $\beta$-arrestin-biased agonists. By contrast, agonists that activate both pathways are designated as balanced agonists. We have recently shown that while compound 48/80 (C48/80) acts as a balanced agonist for MRGPRX2, an angiogenic host defense peptide serves as a $G$ protein-biased agonist (37). The purpose of this study was to test if murepavadin activates human MCs via MRGPRX2 and to determine if it serves as a balanced or biased agonist for the receptor. The data presented herein demonstrate that murepavadin activates human and murine MCs via MRGPRX2 and MrgprB2, respectively and that it serves as a $G$ protein-biased agonist for MRGPRX2 without the involvement of $\beta$-arrestin-mediated receptor regulation. These findings have important implications for the potential utilization of murepavadin in modulating antibiotic-resistant cutaneous infections.

\section{MATERIALS AND METHODS}

\section{Materials}

All reagents used for cell culture were purchased from Invitrogen (Gaithersburg, MD, USA). Recombinant mouse interleukin-3 (IL-3), mouse stem cell factor (SCF), and recombinant human SCF (rhSCF) were obtained from Peprotech (Rocky Hill, NJ, USA). Compound 48/80 (C48/80) was obtained from AnaSpec (Fremont, CA, USA). Murepavadin (Catalog HY-P1674A) was from MedChem Express. P-nitrophenyl- $\mathrm{N}$-acetyl- $\beta$-D-glucosamine (PNAG) was purchased from Sigma-Aldrich (St. Louis, MO, USA). Pertussis toxin (PTx) was from List Biological Laboratories 
(Campbell, CA, USA). Fura-2 acetoxymethyl ester was from Abcam (Cambridge, MA, USA). Bright-Glo Luciferase was from Promega (Madison, WI, USA). Phycoerythrin (PE)-conjugated anti-human MRGPRX2 antibody was from BioLegend (San Diego, CA, USA). Amaxa Nucleofector Kit V was from Lonza (Gaithersburg, MD, USA). DuoSet ELISA kits were from R\&D Systems (Minneapolis, MN, USA). Hemagglutinin (HA)-tagged MRGPRX2 plasmid in pReceiver-MO6 vector was obtained from GeneCopoeia (Rockville, MD, USA). MRGPRX2-Tango plasmid (Addgene no. 66440) was a gift from Dr. Bryan Roth.

\section{Mice}

C57BL/6 (wild-type; WT) mice were purchased from the Jackson Laboratory (Bar Harbor, ME, USA) and housed in pathogen-free cages. WT mice with the deletion of MrgprB2 transcript (MrgprB2 $2^{-/-}$mice) were generated as previously described (38). Eight-to-twelve-week-old male and female mice were used. All animal experiments performed in this study were approved by the Institutional Animal Care and Use Committee of the University of Pennsylvania.

\section{Cell Culture}

The human MC line (LAD2 cell) was kindly provided by Drs. Kirshenbaum and Metcalfe (Laboratory of Allergic Diseases, National Institute of Allergy and Infectious Diseases, National Institutes of Health (NIH), USA). LAD2 cells were cultured in complete StemPro-34 medium supplemented with l-glutamine (2 $\mathrm{mM})$, penicillin $(100 \mathrm{IU} / \mathrm{mL})$, streptomycin $(100 \mu \mathrm{g} / \mathrm{mL})$, and $\mathrm{rhSCF}$ (100 ng/mL), and the medium was hemi-depleted weekly (39).

Rat basophilic leukemia (RBL-2H3) cells were cultured as monolayers in Dulbecco's modified Eagle's medium (DMEM) supplemented with $10 \%$ FBS, L-glutamine $(2 \mathrm{mM})$, penicillin (100 IU/mL) and streptomycin $(100 \mu \mathrm{g} / \mathrm{mL})$ (40). RBL cells stably expressing human MRGPRX2 were maintained similarly in the presence of $1 \mathrm{mg} / \mathrm{mL}$ G418. HTLA cells (HEK-293T cells stably expressing a tTA-dependent luciferase reporter and a $\beta$ arrestin2-TEV protease fusion gene) were cultured in DMEM supplemented with $10 \% \mathrm{FBS}$, L-glutamine $(2 \mathrm{mM})$, penicillin (100 IU/mL), streptomycin $(100 \mu \mathrm{g} / \mathrm{mL})$, hygromycin $(200 \mu \mathrm{g} / \mathrm{mL})$, puromycin $(5 \mu \mathrm{g} / \mathrm{mL})$, and $\mathrm{G} 418(500 \mu \mathrm{g} / \mathrm{mL})$ (41). All cell cultures were kept at $37^{\circ} \mathrm{C}$ incubator with $5 \% \mathrm{CO}_{2}$.

Peritoneal mast cells (PMCs) were established from peritoneal lavages of WT and $\mathrm{MrgprB}^{-1-}$ mice and were cultured for 4-8 weeks in Iscove's Modified Dulbecco's Medium (IMDM) supplemented with $10 \%$ FCS, and recombinant mouse IL-3 (10 ng/mL) and SCF (30 ng/mL). Cells were then determined for $\mathrm{MC}$ receptor expression and function, and were used within $4-8$ weeks $(38,42)$.

\section{Degranulation}

Human LAD2 (1 x $10^{4}$ cells/well), RBL-2H3, RBL-MRGPRX2 $\left(5 \times 10^{4}\right.$ cells/well $)$, or murine peritoneal MCs $\left(5 \times 10^{3}\right.$ cells/well $)$ were washed and plated in a total volume of $50 \mu \mathrm{L}$ HEPES buffer in 96-well plates. Cells were then stimulated with murepavadin for $30 \mathrm{~min}$ at $37^{\circ} \mathrm{C}$. Total level of $\beta$-hexosaminidase release were assessed by lysing the cells with $0.1 \%$ Triton X-100, whereas cells without any stimulation were designated as controls. Aliquots
$(20 \mu \mathrm{L})$ of supernatants were incubated with $1 \mathrm{mM}$ PNAG $(20$ $\mu \mathrm{L})$ for $1 \mathrm{~h}$ at $37^{\circ} \mathrm{C}$. The reaction was then stopped by adding stop buffer $\left.\left(250 \mu \mathrm{L} ; 0.1 \mathrm{M} \mathrm{Na} \mathrm{CO}_{3} / 0.1 \mathrm{M} \mathrm{NaHCO}\right)_{3}\right)$. Quantification of $\beta$-hexosaminidase level was determined by measuring the absorbance at $405 \mathrm{~nm}$ using a Versamax microplate spectrophotometer (Molecular Devices, San Jose, CA, USA) (40).

In some experiments, cells were pretreated with PTx (100 ng/ $\mathrm{mL}, 16 \mathrm{~h}$ ) prior to any stimulation to assess the inhibitory effect of PTx on MC degranulation.

\section{Calcium Mobilization}

Human LAD2 cells $\left(3 \times 10^{5}\right)$, RBL-2H3 or RBL-MRGPRX2 $(2 \times$ $\left.10^{6}\right)$ were loaded with Fura- 2 acetoxymethyl ester $(1 \mu \mathrm{M}$ for $30 \mathrm{~min}$ at $37^{\circ} \mathrm{C}$ ) in $1.5 \mathrm{~mL}$ of HEPES buffer containing $0.1 \%$ BSA. Cells were then washed and allowed complete de-esterification for $15 \mathrm{~min}$ at room temperature. Cells were then resuspended in buffer and stimulated with Murepavadin or C48/80. Calcium mobilization was determined by the ratio between dual excitation wavelengths of 340 and $380 \mathrm{~nm}$, and an emission wavelength of $510 \mathrm{~nm}$ using a Hitachi F-2700 Fluorescence Spectrophotometer.

\section{Cytokine and Chemokine Production and Measurement}

LAD2 cells $\left(3 \times 10^{5}\right.$ cells $\left./ \mathrm{mL}\right)$ were washed with medium, resuspended in fresh medium, and stimulated with indicated concentrations of Murepavadin for $24 \mathrm{~h}$ at $37^{\circ} \mathrm{C}$ with $5 \% \mathrm{CO}_{2}$. Cell-free supernatants were collected and kept at $-80^{\circ} \mathrm{C}$ until further analyses. Similarly, RBL-2H3 or RBL-MRGPRX2 cells $\left(2 \times 10^{5}\right.$ cells $\left./ \mathrm{mL}\right)$ were seeded in a 24 -well plate and cultured overnight in a $37^{\circ} \mathrm{C}$ incubator with $5 \% \mathrm{CO}_{2}$. The next day, the medium was aspirated, fresh medium was added to the cells, and the cells were stimulated with indicated concentrations of Murepavadin for $24 \mathrm{~h}$ at $37^{\circ} \mathrm{C}$ with $5 \% \mathrm{CO}_{2}$. Cell-free supernatants were collected and kept at $-80^{\circ} \mathrm{C}$ until further analyses. The cytokine and chemokine production were measured using human CCL3/MIP-1 alpha, human IL-8/ CXCL8, rat JE/MCP-1/CCL2 and rat TNF-alpha DuoSet ELISA kits (R\&D Systems) following the manufacturer's protocols.

To determine the inhibitory effect of PTx on cytokine and chemokine production, pretreatment with PTx $(100 \mathrm{ng} / \mathrm{mL}$, $16 \mathrm{~h}$ ) was performed prior to any stimulation.

\section{Generation of Cells Transiently Expressing MRGPRX2 and Its Variants}

Transient transfections in RBL-2H3 cells expressing WTMRGPRX2 and its naturally occurring missense variants within MRGPRX2's ligand binding cradle (G165E) or G protein-coupling region (V282M) were performed as described previously $(43,44)$. RBL-2H3 cells $\left(2 \times 10^{6}\right)$ were transfected with $2 \mu \mathrm{g}$ of HA-tagged plasmid using the Amaxa Nucleofector Device and Amaxa Kit V and were used within 16 - $20 \mathrm{~h}$ post-transfection.

Flow cytometry was used to determine cell surface expression of transiently transfectants. Cells $\left(5 \times 10^{5}\right)$ were incubated with PE- 
anti-MRGPRX2 antibody for $30 \mathrm{~min}$, washed in ice-cold FACS buffer (PBS containing 2\% FCS and $0.09 \% \mathrm{NaN}_{3}$ ), followed by fixation with $1.5 \%$ paraformaldehyde. Expression level of MRGPRX2 and its variants were analyzed using a BD LSR II flow cytometer (San Jose, CA, USA) with WinList software, version 8.

\section{Transcriptional Activation Following Arrestin Translocation (Tango) Assay}

HTLA-MRGPRX2 cells $\left(5 \times 10^{4}\right.$ cells/well, 96-well plate $)$ were cultured overnight in a $37^{\circ} \mathrm{C}$ incubator. The next day, the medium was removed and cells were exposed to Murepavadin in an antibiotic-free medium $(160 \mu \mathrm{L})$ for $16 \mathrm{~h}$ at $37^{\circ} \mathrm{C}$. The medium was then replaced with $100 \mu \mathrm{L}$ of Bright-Glo solution and relative luminescence was analyzed using a Thermo Luminoskan Ascent 392 Microplate Luminometer (41).

\section{Receptor Internalization}

RBL-MRGPRX2 or HTLA-MRGPRX2 cells $\left(5 \times 10^{5}\right)$ were treated with C48/80 or Murepavadin for $30 \mathrm{~min}, 3,6$, and $16 \mathrm{~h}$ to induce cell surface receptor internalization. After indicated time, cell surface expression was determined by flow cytometry as described above.

\section{Evan's Blue Dye Extravasation}

Mice (WT and $\mathrm{MrgprB2}^{-/-}$) were intravenously injected with $1 \%$ Evan's blue followed by intradermal injection of $20 \mu \mathrm{L}$ Murepavadin $(30 \mu \mathrm{M})$ in the right paw and PBS (vehicle) in the left paw. After $30 \mathrm{~min}$, the mice were euthanized and the paws were removed, weighed, dissolved in $500 \mu \mathrm{L}$ formamide and incubated at $56^{\circ} \mathrm{C}$ overnight. The Evan's blue dye extravasation was measured by collecting the supernatants and the absorbance was measured at 650 $\mathrm{nm}$ using microplate spectrophotometer.

\section{Statistical Analysis}

Data shown represent mean \pm SEM value derived from at least three independent experiments. Statistical significance was calculated using $t$-test and one-way or two-way ANOVA analyzed by GraphPad Prism version 9.0.1. Significant differences were set at ${ }^{\star} p<0.05,{ }^{* *} p<0.01,{ }^{* *} p<0.001,{ }^{* * *} p<0.0001$.

\section{RESULTS}

\section{Murepavadin Induces Intracellular $\mathrm{Cas}^{2+}$ Mobilization, Degranulation and Chemokine Generation in Human MCs}

We previously demonstrated that LL-37, human $\beta$-defensins and protegrin-1 activate human MCs via MRGPRX2 at concentrations ranging from $1-10 \mu \mathrm{M}(22,23,29)$. To determine if murepavadin activates MCs, we initially utilized a human MC line, LAD2 cells and tested the ability of $10 \mu \mathrm{M}$ murepavadin to induce $\mathrm{Ca}^{2+}$ mobilization. As shown in Figure 1A, murepavadin at this concentration induced a robust and sustained $\mathrm{Ca}^{2+}$ response. Next, we asked if murepavadin induces MC degranulation. We found that murepavadin induced a dose-dependent degranulation as measured by $\beta$-hexosaminidase release, reaching $\sim 80 \%$ at a concentration of $10 \mu \mathrm{M}$ with an $\mathrm{EC}_{50}$ value of $\sim 3 \mu \mathrm{M}$ (Figure 1B). While MC degranulation promotes increased vascular permeability in vivo, its innate and adaptive immune function require the generation of chemokines. We therefore investigated the ability of murepavadin to stimulate chemokine IL-8 and CCL3 production in LAD2 cells. At a concentration of $10 \mu \mathrm{M}$, murepavadin induced substantial IL-8 (Figure 1C) and CCL3 (Figure 1D).

To explore the underlying mechanism via which murepavadin activates MCs, we utilized RBL-2H3 cells that were transfected to stably express human MRGPRX2 (RBLMRGPRX2). We found that murepavadin induced $\mathrm{Ca}^{2+}$ mobilization, $\beta$-hexosaminidase as well as TNF- $\alpha$ and CCL2 production from RBL-MRGPRX2, but not from untransfected cells (Figures 2A-E). Moreover, we found that Pertussis toxin

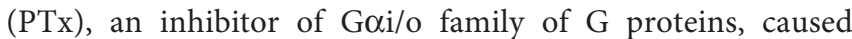
significant inhibition of murepavadin-induced degranulation, TNF- $\alpha$ and CCL2 production (Figures 2C-E). Taken together, these findings demonstrate that murepavadin causes the activation of human MCs specifically via MRGPRX2 and that these responses are $\mathrm{G}$ protein-dependent.

\section{Naturally Occurring MRGPRX2 Missense Variants Are Hypo-Responsive to Murepavadin for MC Degranulation}

We have previously identified naturally occurring loss-offunction MRGPRX2 missense variants within the receptor's ligand binding (G165E) and G protein coupling (V282M) domains (Figures 3A, B) $(43,44)$. To further validate
A

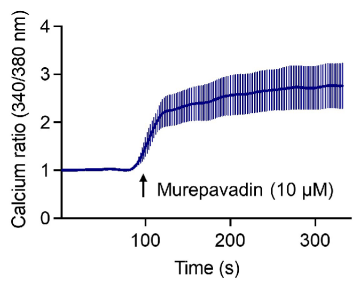

B

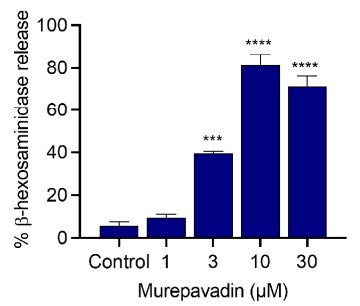

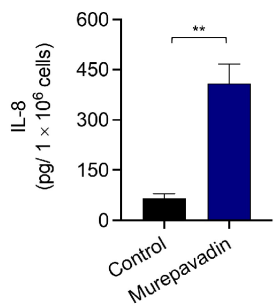

D

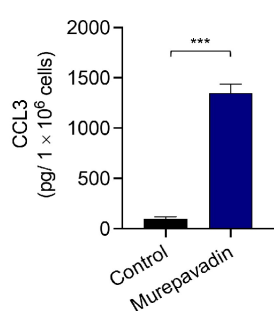

FIGURE 1 | Murepavadin induces intracellular $\mathrm{Ca}^{2+}$ mobilization, degranulation and causes chemokine production in human MCs. (A) $\mathrm{Ca}^{2+}$ mobilization measurement following murepavadin $(10 \mu \mathrm{M})$ stimulation of Fura-2-loaded LAD2 cells. (B) LAD2 cells were exposed to indicated concentrations of murepavadin (30 $\mathrm{min}$ ) and degranulation was assayed by measuring the release of $\beta$-hexosaminidase. (C, D) LAD2 cells were exposed to $10 \mu \mathrm{M}$ of murepavadin for $24 \mathrm{~h}$ and the production of IL-8 and CCL3 were determined by ELISA. Data presented are the mean \pm SEM of at least three experiments. Statistical significance was determined by $t$-test or one-way ANOVA with Dunnett's multiple comparisons at a value ${ }^{* \star} p<0.01,{ }^{* \star *} p<$ 0.001 , and ${ }^{* \star \star *} p<0.0001$. 


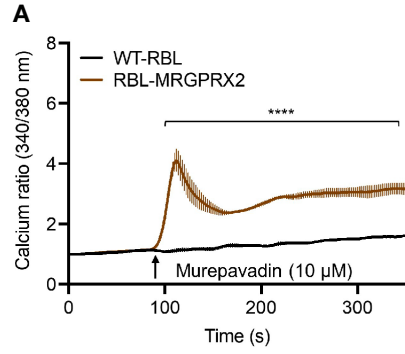

D

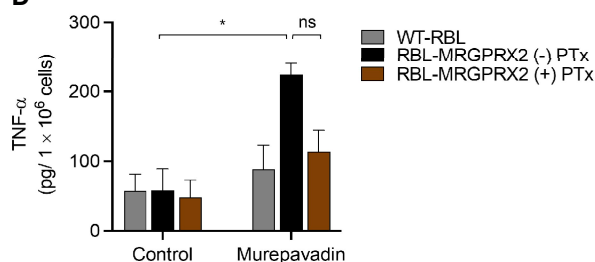

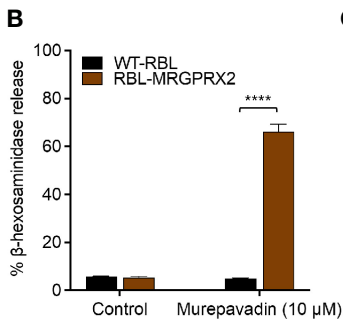

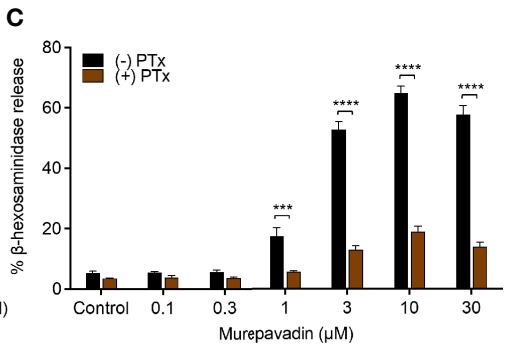

E

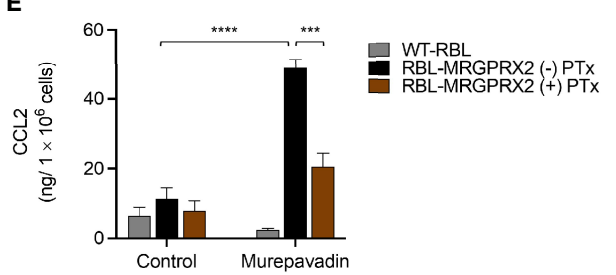

FIGURE 2 | Murepavadin induces intracellular $\mathrm{Ca}^{2+}$ mobilization, degranulation and chemokine production in MCs through MRGPRX2. (A) Calcium mobilization measurement following murepavadin $(10 \mu \mathrm{M})$ stimulation of Fura-2 loaded WT-RBL and RBL-MRGPRX2 cells. (B) WT-RBL and RBL-MRGPRX2 cells were exposed to $10 \mu \mathrm{M}$ murepavadin (30 min), and degranulation was assayed by measuring the release of $\beta$-hexosaminidase. (C) RBL-MRGPRX2 cells were incubated in the presence or absence of pertussis toxin (PTx), at a concentration of $100 \mathrm{ng} / \mathrm{mL}$ for $16 \mathrm{~h}$ and exposed to indicated concentrations of murepavadin (30 min) and degranulation was assayed by measuring the release of $\beta$-hexosaminidase. (D, E) WT-RBL and RBL-MRGPRX2 cells were incubated in the presence or absence of pertussis toxin (PTx) at a concentration of $100 \mathrm{ng} / \mathrm{mL}$ for $16 \mathrm{~h}$ and exposed to $10 \mu \mathrm{M}$ of murepavadin $(24 \mathrm{~h})$ and the production of cytokine TNF- $\alpha$ and chemokine CCL2 were quantified by ELISA. Data presented are the mean \pm SEM of at least three experiments. Statistical significance was determined by two-way ANOVA with Š́dák's or Tukey's multiple comparisons at a value ${ }^{\star} p<0.05,{ }^{\star \star \star} p<0.001,{ }^{\star \star \star \star} p<0.0001$, and ns denotes "not significant".

murepavadin's specificity for MRGPRX2, we transiently transfected RBL-2H3 cells with cDNAs encoding each of these variants. Flow cytometry analyses confirmed cell surface expression of MRGPRX2 (WT) and its missense variants (G165E and V282M) (Figure 3C). While murepavadin $(10 \mu \mathrm{M})$ induced $\beta$-hexosaminidase release in cells expressing WT-MRGPRX2, this response was significantly inhibited in cells transiently expressing the G165E or V282M variant (Figure 3D). These findings substantiate the notion that murepavadin utilizes MRGPRX2 to activate MCs.

\section{Murepavadin Does Not Promote $\beta$-Arrestin Recruitment Following MRGPRX2 Activation}

To determine if murepavadin promotes $\beta$-arrestin recruitment in addition to G proteins, we utilized HTLA (HEK-293T cells that were transfected with a tTA-dependent luciferase reporter and a $\beta$-arrestin2-TEV protease fusion gene) cells stably expressing MRGPRX2 (HTLA-MRGPRX2). We have previously shown that C48/80 strongly promotes $\beta$-arrestin recruitment in HTLAMRGPRX2 cells as determined by a transcriptional activation following arrestin translocation (Tango) assay (37). We therefore utilized C48/80 as a positive control and tested the ability of murepavadin to promote $\beta$-arrestin recruitment. As shown in Figures 4A, B, while C48/80 $(3 \mu \mathrm{g} / \mathrm{mL})$ induced $\sim 30$-fold increase in $\beta$-arrestin-mediated gene expression when compared to buffer control, murepavadin was without effect.

\section{Murepavadin Does Not Induce MRGPRX2 Internalization or Desensitization}

We have previously shown that $\mathrm{C} 48 / 80$ not only induces $\beta$ arrestin recruitment but also causes MRGPRX2 internalization (37). We found that incubation of HTLA-MRGPRX2 cells with C48/80 (3 $\mu \mathrm{g} / \mathrm{mL} ; 0.5 \mathrm{~h}-16 \mathrm{~h})$ induced substantial receptor internalization but murepavadin had no effect (Figures 4C, D). The data presented in Figures 4E, $F$ show representative histograms of cell surface MRGPRX2 following incubation of HTLA-MRGPRX2 cells with C48/80 (3 $\mu \mathrm{g} / \mathrm{mL})$ and murepavadin $(10 \mu \mathrm{M})$ for $16 \mathrm{~h}$. These data clearly demonstrate that while $C 48 / 80$ promotes $\beta$-arrestin recruitment and caused MRGPRX2 internalization, murepavadin does not induce these responses.

To confirm the biological relevance of the findings described above, we performed receptor internalization studies in RBLMRGPRX2 cells by flow cytometry. Similar to the situation in HTLA-MRGPRX2 cells, C48/80 induced substantial receptor internalization in RBL-MRGPRX2 cells but murepavadin had no effect (Figures 5A, B). As for $\beta$-arrestin recruitment (Figure 4A) and receptor internalization (Figures 5A, B), incubation of cells with $\mathrm{C} 48 / 80(3 \mu \mathrm{g} / \mathrm{mL}, 16 \mathrm{~h})$ resulted in substantial inhibition of $\mathrm{Ca}^{2+}$ mobilization response to the stimulation by the same agonist (Figure 5C). Conversely, cells preincubated with murepavadin $(10 \mu \mathrm{M}, 16 \mathrm{~h})$ had little to no effect on $\mathrm{Ca}^{2+}$ response to stimulation by the same agonist (Figure 5D). 
A

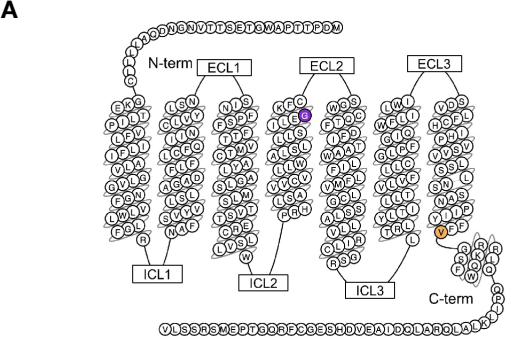

C

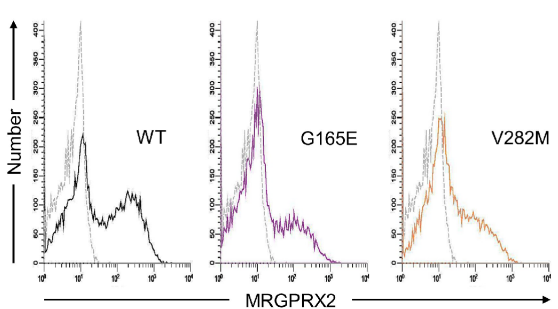

\begin{tabular}{|ccccc|}
\hline $\begin{array}{c}\text { Protein } \\
\text { Change }\end{array}$ & $\begin{array}{c}\text { Number } \\
\text { GPCRdb }\end{array}$ & $\begin{array}{c}\text { Functional } \\
\text { Annotation }\end{array}$ & Rs Number \\
\hline 1 & G165E & $4.61 \times 61$ & Ligand binding & rs 141744602 \\
\hline 2 & V282M & $7.56 \times 56$ & G protein coupling & rs 779414608 \\
\hline
\end{tabular}

D

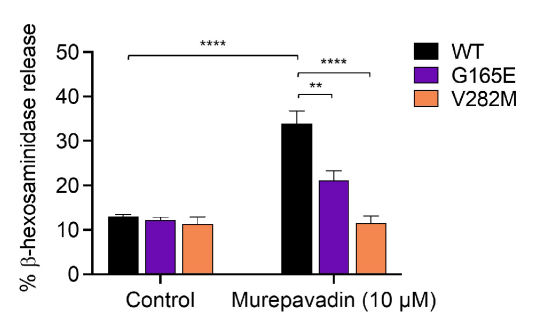

FIGURE 3 | Naturally occurring MRGPRX2 missense variants are hypo-responsive to murepavadin for MC degranulation. (A) Snake diagram of MRGPRX2 indicating the two amino acid residues to be investigated. (B) Single amino acid substitution for each of the MRGPRX2 variant is shown in the table. (C) Cell surface receptor expression of the wild-type MRGPRX2 (WT) and its missense variants (G165E and V282M) was confirmed using flow cytometry. (D) RBL cells expressing MRGPRX2 (WT) and its variants (G165E and V282M) were exposed to $10 \mu \mathrm{M}$ murepavadin (30 min) and degranulation was assayed by measuring the release of $\beta$-hexosaminidase. Statistical significance was determined by two-way ANOVA with Tukey's multiple comparisons at a value ${ }^{\star \star} p<0.01$ and ${ }^{\star \star \star *} p<0.0001$.

\section{Murepavadin Activates Murine MCs In Vitro and In Vivo via MrgprB2}

It has been previously shown that C48/80 causes substantial degranulation in mouse peritoneal MCs (PMCs) via MrgprB2 (21). To test if murepavadin induces degranulation in murine
PMCs and to determine the role of MrgprB2 in this response, we cultured PMCs from peritoneal lavage of wild-type (WT) and $\mathrm{MrgprB}^{-1-}$ mice $(38,42)$. We found that murepavadin induced degranulation in PMCs cultured from WT mice but this response was not observed in cells cultured from $\mathrm{MrgprB}^{-/-}$mice

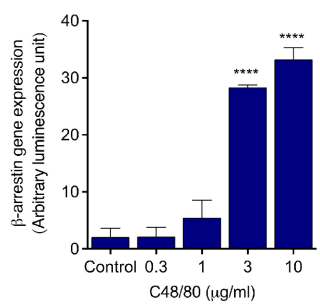

B

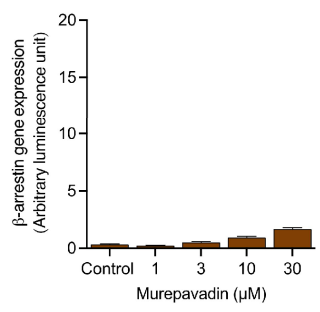

C

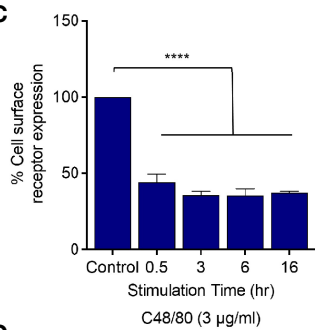

D

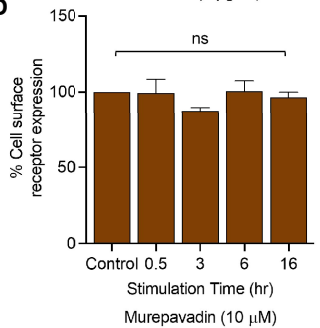

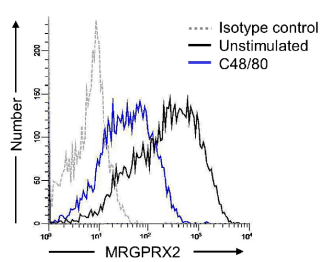

$\mathbf{F}$

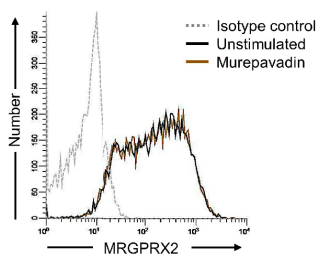

FIGURE 4 | Murepavadin does not promote $\beta$-arrestin recruitment following MRGPRX2 activation. (A, B) HTLA-MRGPRX2 cells were exposed to indicated concentrations of C48/80 or murepavadin for $16 \mathrm{~h}$. Medium was removed and Bright-Glo solution (100 $\mu \mathrm{L})$ was added into each well (96-well plate) and $\beta$-arrestin gene expression (in relative luminescence unit) was measured. (C, D) HTLA-MRGPRX2 cells were exposed to MRGPRX2 ligand (C48/80 or murepavadin, 16 h) and cell surface receptor expression of MRGPRX2 was confirmed using flow cytometry and quantified using a mean fluorescent intensity (MFI) in comparison to the untreated control. (E, F) Representative histograms of MRGPRX2 cell surface receptor expression of HTLA-MRGPRX2 cells. Statistical significance was determined by one-way ANOVA with Dunnett's multiple comparisons at a value ${ }^{\star \star \star \star} p<0.0001$ and ns denotes "not significant". 
A

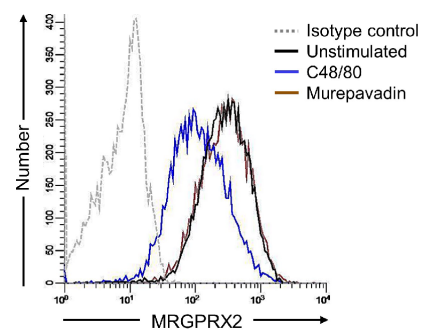

B

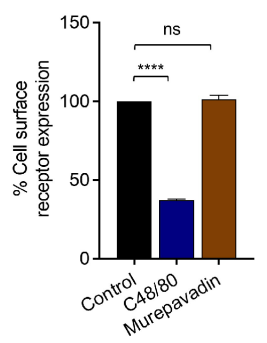

C

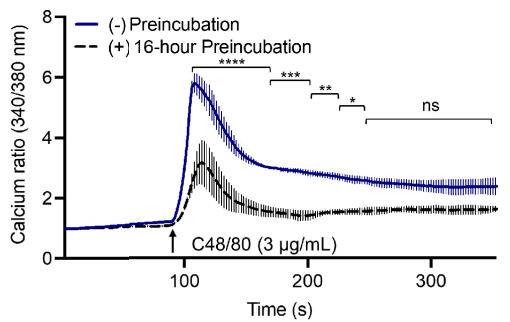

D

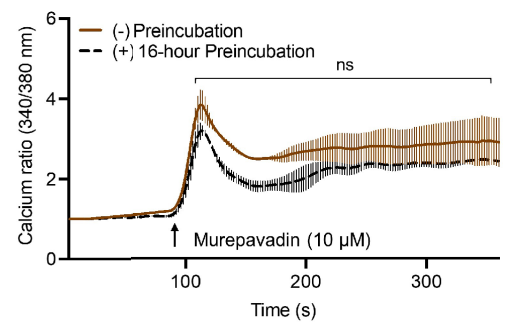

FIGURE 5 | Murepavadin does not induce MRGPRX2 internalization or desensitization. (A) RBL-MRGPRX2 cells were exposed to MRGPRX2 ligand (C48/80 3 4 g/ $\mathrm{mL}$ or murepavadin $10 \mu \mathrm{M}, 16 \mathrm{~h}$ ) and cell surface receptor expression of MRGPRX2 was confirmed using flow cytometry. A representative histogram of MRGPRX2 cell surface receptor expression is shown. (B) Quantitative analysis of cell surface receptor expression was calculated using a mean fluorescent intensity (MFI) in comparison to the untreated control. (C, D) Calcium mobilization measurements of RBL-MRGPRX2 cells exposed to MRGPRX2 ligand (C48/80 $3 \mu \mathrm{g} / \mathrm{mL}$ or murepavadin $10 \mu \mathrm{M}, 16 \mathrm{~h})$ following C48/80 (3 $\mu \mathrm{g} / \mathrm{mL})$ or murepavadin (10 $\mu \mathrm{M})$ stimulation, respectively. Statistical significance was determined by one-way ANOVA with Dunnett's multiple comparisons and two-way ANOVA with Šídák's multiple comparisons at a value ${ }^{\star} p<0.05,{ }^{\star \star} p<0.01,{ }^{\star \star \star} p<0.001$, ${ }^{\star \star \star *} p<0.0001$, and ns denotes "not significant".

(Figure 6A). To determine if murepavadin induces degranulation of cutaneous $\mathrm{MC}$ in vivo, we performed intradermal injection of murepavadin $(30 \mu \mathrm{M}, 20 \mu \mathrm{L})$ or PBS into the paw after intravenous injection of Evan's blue dye. Consistent with peritoneal MC degranulation in vitro, murepavadin caused a significant increase in vascular permeability when compared to PBS. However, this vascular permeability response was abolished in $\mathrm{MrgprB}^{-/-}$mice (Figures 6B, C). Together, these data demonstrate that murepavadin induces degranulation in murine MCs to cause increased vascular permeability via the activation of MrgprB2.

\section{DISCUSSION}

Murepavadin is a synthetic cyclic $\beta$-hairpin HDP mimetic that targets an outer membrane protein transporter $\mathrm{LptD}$ of the Gram-

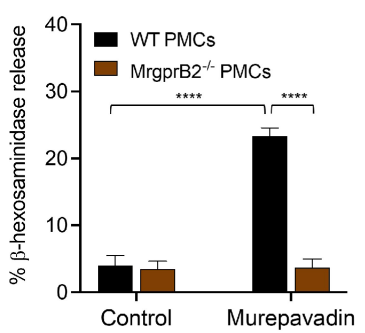

B WT

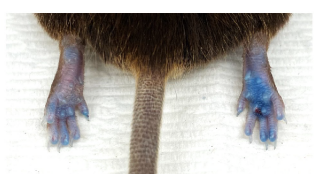

Murepavadin

MrgprB2--
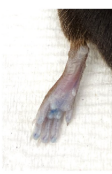

Vehicle

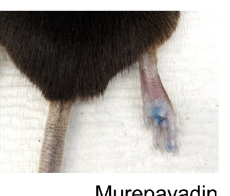

Murepavadin
C

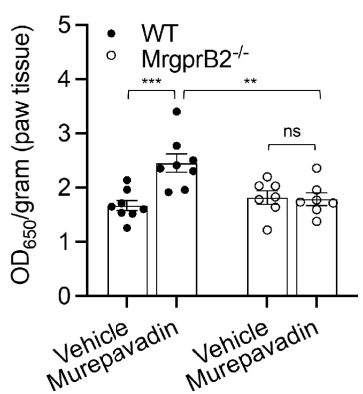

FIGURE 6 | Murepavadin activates murine MCs in vitro and in vivo via MrgprB2. (A) WT and MrgprB2 ${ }^{-/-}$PMCs were exposed to $10 \mu \mathrm{M}$ murepavadin (30 min) and degranulation was assayed by measuring the release of $\beta$-hexosaminidase. (B) Representative images of Evan's blue dye extravasation in WT and MrgprB2 ${ }^{-1-}$ mice in vivo. Mice were intradermally injected with $20 \mu \mathrm{L}$ of murepavadin (30 $\mu \mathrm{M})$ or PBS, and extravasation of Evan's blue dye was determined after 30 min. (C) Quantification of extravasation of Evan's blue dye in WT and MrgprB2 $2^{-/-}$mice $(n=7-8)$. Statistical significance was determined by two-way ANOVA with Tukey's multiple comparisons at a value ${ }^{* \star} p<0.01,{ }^{* \star *} p<0.001,{ }^{* \star \star *} p<0.0001$, and ns denotes "not significant". 
negative bacterium $P$. aeruginosa, which makes it highly specific to this pathogen $(45,46)$. In in vitro studies, murepavadin is very effective against a wide-ranging species of multi-drug resistant Pseudomonas bacteria and demonstrates exceptional efficacy in sepsis, lung, and thigh infection models in vivo (47). However, intravenous administration of murepavadin for treating nosocomial pneumonia has been temporarily halted due to reports of kidney injury. Despite this, an inhaled formulation of murepavadin is under investigation for its potential effectiveness in treating Pseudomonas infection in patients with cystic fibrosis $(33,48)$. We made the novel observation that murepavadin is able to induce human MC activation through MRGPRX2 and murine MC activation through MrgprB2. Because human skin MCs express MRGPRX2 at high levels $(17,19,49)$, these findings suggest that murepavadin can be utilized for treating $P$. aeruginosa skin infection through harnessing MC's host defense and wound healing properties.

$P$. aeruginosa skin infection is associated with high morbidity and mortality rates mainly because of its ability to form biofilms and to resist multiple antibiotics $(25,26)$. Therefore, novel treatment approach together with rapid wound closure are critical to control this type of infection. Weller et al. (50), showed that skin wounding in mice results in MC degranulation. This, in turn, causes increased vascular permeability and neutrophil recruitment. Using the same wound model but superimposed with $P$. aeruginosa, Zimmerman et al. (27), showed that MCs are essential in controlling bacterial infection and promoting healing. It was proposed that IL-1 and IL33 generated by keratinocytes in response to infection activate MCs to produce IL-6, which in turn generate HDPs from keratinocytes resulting in the direct bacterial killing. However, recent studies demonstrated that HDPs can also induce MC degranulation in human (via MRGPRX2) and mouse (via MrgprB2) (22-24). Based on these findings, we suspect that the activation of MCs via MRGPRX2/B2 confer protective immunity and contribute to host defense. The minimum concentration of murepavadin required to inhibit $90 \%$ growth of $P$. aeruginosa $\left(\mathrm{MIC}_{90}\right.$ ) was reported to be in the range of $2-32 \mathrm{mg} / \mathrm{L}(33,48)$. We found that murepavadin ( $1 \mu \mathrm{M} ; 1.667 \mathrm{mg} / \mathrm{L}$ ) induced significant MC degranulation via MRGPRX2 and that maximal response was obtained at a concentration of $10 \mu \mathrm{M}(16.67 \mathrm{mg} / \mathrm{L})$. Murepavadin at $10 \mu \mathrm{M}$, which is relevant for direct inhibition of $P$. aeruginosa growth, induced TNF- $\alpha$, IL- 8 and CCL3 production from MCs via MRGPRX2. A topical application of murepavadin is currently under investigation for treating $P$. aeruginosa cutaneous infection mainly because of its direct and specific activity against the pathogen $(34,35)$. Based on the data presented herein, we propose that potential effectiveness of murepavadin also reflects its ability to harness MC's immunomodulatory property via the activation of MRGPRX2 (Figure 7).

It is well documented that Staphylococcus aureus is responsible for the majority of bacterial skin infections $(51,52)$. In a murine model of $S$. aureus skin infection, topical application of mastoparan, an amphipathic peptide found in Hymenoptera venom that causes MC activation via MrgprB2, results in the recruitment of immune cells particularly neutrophils, This contributes to subsequent bacterial clearance (15). However, a mastoparan derivative that does not induce MC degranulation is unable to control S. aureus infection despite the fact that it demonstrates direct antibacterial activity. By contrast, another mastoparan derivative that induces MC degranulation via MrgprB2 but has no direct antibacterial activity effectively controls infection. These findings suggest that the potential therapeutic effectiveness of mastoparan could reflect its action on MCs via MRGPRX2 rather than its direct antibacterial activity.

Apart from degranulation, mastoparan also triggers the generation of chemokines CCL2, CCL3 and CCL4 from MCs, which promote the mobilization of dendritic cells (DCs)

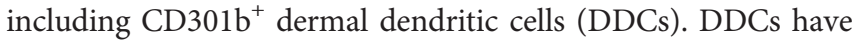
been implicated in promoting re-epithelialization of sterile wounds and accelerating wound closure. Depletion of MrgprB2-expressing MCs results in significantly decreased number of DDCs, suggesting that MCs contribute to the restoration of DDCs population to homeostatic levels, thus contributing to regenerative healing (15). Moreover, mastoparan also contributes to the production of $S$. aureusspecific IgG following initial S. aureus skin infection, which is associated with reduced lesion size during subsequent bacterial reinfection (15). Thus, it is highly likely that activation of murine MCs by mastoparan through MrgprB2 confers both innate and adaptive immunity to provide protection against bacterial infection and reinfection. Given that murepavadin activates human MCs via MRGPRX2, it should be possible to use this HDP mimetic for the modulation of $S$. aureus skin infection. Because murepavadin appears to have a lower $\mathrm{EC}_{50}$ value for $\mathrm{MC}$ degranulation than mastoparan (15), our prediction is that lower concentration of the drug will be required to treat $S$. aureus infection. Also, since murepavadin is an HDP mimetic and is less susceptible to degradation than mastoparan, its effectiveness is likely to be greater than that of mastoparan, thus requiring fewer treatments to clear infection and to promote healing.

It is generally accepted that most GPCRs activate two parallel but independent signaling pathways; one involving $G$ proteins while second pathway is independent of $G$ proteins but requires the recruitment of $\beta$-arrestins (53-56). For certain GPCRs, agonists can activate only $G$ proteins ( $G$ protein biased) or only $\beta$-arrestin ( $\beta$ arrestin biased) or both (balanced). Agonists that induce both pathways not only activate $G$ protein-mediated signaling but also result in dissociation of $G$ proteins from the receptor (desensitization) and promote the receptor internalization. We have previously shown that while $\mathrm{C} 48 / 80$ acts as balanced agonist for MRGPRX2, an HDP angiogenic peptide, AG-30/5C acts as a G protein-biased agonist for the receptor. Thus, C48/80 caused substantial $\beta$-arrestin recruitment while AG-30/5C had little to no effect. Most importantly, preincubation of cells with $\mathrm{C} 48 / 80$ resulted in a significant reduction of cell surface receptor expression and loss of cell responsiveness to all MRGPRX2 agonists tested. By contrast, AG-30/5C had little to no effect on cell surface receptor expression or MC degranulation to any of the MRGPRX2 agonists tested (37). The data presented herein demonstrate that similar to AG-30/5C, murepavadin acts as a G protein-biased agonist for MRGPRX2. Thus, it is possible that the resistance MRGPRX2 to undergo desensitization and internalization by murepavadin could enhance its therapeutic potential. 


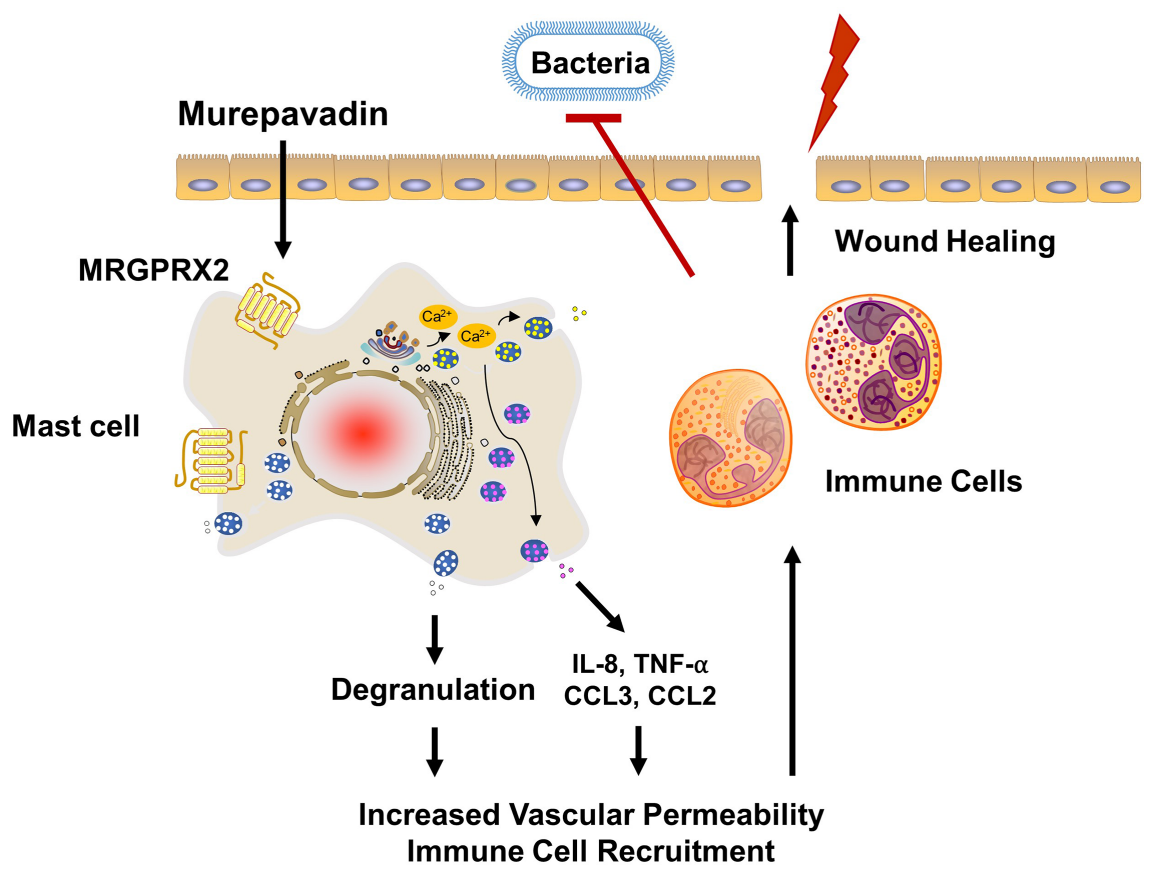

FIGURE 7 | Proposed mechanism of action of murepavadin/MRGPRX2-induced bacterial clearance and wound healing. Murepavadin activates MCs via MRGPRX2. An increase in intracellular calcium following murepavadin stimulation triggers MC degranulation and chemokine production (IL-8, CCL3, CCL2, and TNF- $\alpha$ ). These MC mediators induce immune cell recruitment and subsequently contribute to host defense and wound healing.

In summary, although HDPs have therapeutic potential for the treatment of multi-drug-resistant bacterial infections, their relative instability and cytotoxicity have limited their usefulness as prospective antimicrobial agents. Small molecule HDP mimetics do not display these limitations and murepavadin was synthesized to specifically target $P$. aeruginosa. However, the possibility that murepavadin could activate MCs has not been suspected. In the present study, we have shown that murepavadin induces degranulation, TNF- $\alpha$, IL- 8 and CCL3 production via MRGPRX2, a receptor that is primarily expressed in human skin MCs. Thus, the ability of murepavadin to exploit MC's immunomodulatory functions could form the basis for the treatment of skin infection caused by bacterial species such as $P$. aeruginosa and $S$. aureus. Furthermore, the findings presented herein that MCs expressing missense MRGPRX2 variants G165E (rs141744602) and V282M (rs779414608) were resistant to murepavadin-induced degranulation likely has important clinical implications. It is possible that murepavadin may not effectively clear microbial infection in individuals harboring these MRGPRX2 polymorphisms because of their inability to support MC degranulation.

\section{DATA AVAILABILITY STATEMENT}

The original contributions presented in the study are included in the article/supplementary material. Further inquiries can be directed to the corresponding author.

\section{ETHICS STATEMENT}

The animal study was reviewed and approved by The Institutional Animal Care and Use Committee of the University of Pennsylvania.

\section{AUTHOR CONTRIBUTIONS}

HA contributed to conception, supervision and funding acquisition of the study. AA and CC performed the experiments and analyzed the data. AA and HA wrote the first draft of the manuscript. All authors contributed to the article and approved the submitted version.

\section{FUNDING}

This work was supported by grants R01-AI143185, R01AI149487 and R01-AI124182 to HA.

\section{ACKNOWLEDGMENTS}

We would like to thank Drs. Arnold Kirshenbaum and Dean Metcalfe (National Institute of Allergy and Infectious Diseases) for providing LAD2 cells. We also thank the FACS core facility of the University of Pennsylvania School of Dental Medicine for flow cytometry acquisition and analysis. 


\section{REFERENCES}

1. Ardal C, Balasegaram M, Laxminarayan R, McAdams D, Outterson K, Rex JH, et al. Antibiotic Development - Economic, Regulatory and Societal Challenges. Nat Rev Microbiol (2020) 18(5):267-74. doi: 10.1038/s41579-019-0293-3

2. Boldenow E, Jones S, Lieberman RW, Chames MC, Aronoff DM, Xi C, et al. Antimicrobial Peptide Response to Group B Streptococcus in Human Extraplacental Membranes in Culture. Placenta (2013) 34(6):480-5. doi: 10.1016/j.placenta.2013.02.010

3. Zaga-Clavellina V, Ruiz M, Flores-Espinosa P, Vega-Sanchez R, Flores-Pliego A, Estrada-Gutierrez G, et al. Tissue-Specific Human Beta-Defensins (HBD)1, HBD-2 and HBD-3 Secretion Profile From Human Amniochorionic Membranes Stimulated With Candida Albicans in a Two-Compartment Tissue Culture System. Reprod Biol Endocrinol (2012) 10:70. doi: 10.1186/ 1477-7827-10-70

4. van der Does AM, Bergman P, Agerberth B, Lindbom L. Induction of the Human Cathelicidin LL-37 as a Novel Treatment Against Bacterial Infections. J Leukoc Biol (2012) 92(4):735-42. doi: 10.1189/jlb.0412178

5. Rohrl J, Yang D, Oppenheim JJ, Hehlgans T. Human Beta-Defensin 2 and 3 and Their Mouse Orthologs Induce Chemotaxis Through Interaction With CCR2. J Immunol (2010) 184(12):6688-94. doi: 10.4049/jimmunol.0903984

6. Yang D, Liu ZH, Tewary P, Chen Q, de la Rosa G, Oppenheim JJ. Defensin Participation in Innate and Adaptive Immunity. Curr Pharm Des (2007) 13 (30):3131-9. doi: 10.2174/138161207782110453

7. Niyonsaba F, Iwabuchi K, Someya A, Hirata M, Matsuda H, Ogawa H, et al. A Cathelicidin Family of Human Antibacterial Peptide LL-37 Induces Mast Cell Chemotaxis. Immunology (2002) 106(1):20-6. doi: 10.1046/j.13652567.2002.01398.x

8. Koczulla R, von Degenfeld G, Kupatt C, Krotz F, Zahler S, Gloe T, et al. An Angiogenic Role for the Human Peptide Antibiotic LL-37/hCAP-18. J Clin Invest (2003) 111(11):1665-72. doi: 10.1172/JCI17545

9. Hirsch T, Spielmann M, Zuhaili B, Fossum M, Metzig M, Koehler T, et al. Human Beta-Defensin-3 Promotes Wound Healing in Infected Diabetic Wounds. J Gene Med (2009) 11(3):220-8. doi: 10.1002/jgm.1287

10. Dawson RM, Liu CQ. Disulphide Bonds of the Peptide Protegrin-1 Are Not Essential for Antimicrobial Activity and Haemolytic Activity. Int J Antimicrob Agents (2010) 36(6):579-80. doi: 10.1016/j.ijantimicag.2010.08.011

11. Westman J, Hansen FC, Olin AI, Morgelin M, Schmidtchen A, Herwald H. p33 (gC1q Receptor) Prevents Cell Damage by Blocking the Cytolytic Activity of Antimicrobial Peptides. J Immunol (2013) 191(11):5714-21. doi: 10.4049/ jimmunol.1300596

12. Ashby M, Petkova A, Hilpert K. Cationic Antimicrobial Peptides as Potential New Therapeutic Agents in Neonates and Children: A Review. Curr Opin Infect Dis (2014) 27(3):258-67. doi: 10.1097/QCO.0000000000000057

13. Hilchie AL, Wuerth K, Hancock RE. Immune Modulation by Multifaceted Cationic Host Defense (Antimicrobial) Peptides. Nat Chem Biol (2013) 9 (12):761-8. doi: 10.1038/nchembio.1393

14. Galli SJ, Gaudenzio N, Tsai M. Mast Cells in Inflammation and Disease: Recent Progress and Ongoing Concerns. Annu Rev Immunol (2020) 38:49-77. doi: 10.1146/annurev-immunol-071719-094903

15. Arifuzzaman M, Mobley YR, Choi HW, Bist P, Salinas CA, Brown ZD, et al. MRGPR-Mediated Activation of Local Mast Cells Clears Cutaneous Bacterial Infection and Protects Against Reinfection. Sci Adv (2019) 5(1):eaav0216. doi: 10.1126/sciadv.aav0216

16. Pundir P, Liu R, Vasavda C, Serhan N, Limjunyawong N, Yee R, et al. A Connective Tissue Mast-Cell-Specific Receptor Detects Bacterial QuorumSensing Molecules and Mediates Antibacterial Immunity. Cell Host Microbe (2019) 26(1):114-22 e8. doi: 10.1016/j.chom.2019.06.003

17. Fujisawa D, Kashiwakura J, Kita H, Kikukawa Y, Fujitani Y, Sasaki-Sakamoto $\mathrm{T}$, et al. Expression of Mas-Related Gene X2 on Mast Cells Is Upregulated in the Skin of Patients With Severe Chronic Urticaria. J Allergy Clin Immunol (2014) 134(3):622-33.e9. doi: 10.1016/j.jaci.2014.05.004

18. Tatemoto K, Nozaki Y, Tsuda R, Konno S, Tomura K, Furuno M, et al. Immunoglobulin E-Independent Activation of Mast Cell Is Mediated by Mrg Receptors. Biochem Biophys Res Commun (2006) 349(4):1322-8. doi: 10.1016/ j.bbrc.2006.08.177

19. Plum T, Wang X, Rettel M, Krijgsveld J, Feyerabend TB, Rodewald HR. Human Mast Cell Proteome Reveals Unique Lineage, Putative Functions, and
Structural Basis for Cell Ablation. Immunity (2020) 52(2):404-16.e5. doi: 10.1016/j.immuni.2020.01.012

20. Motakis E, Guhl S, Ishizu Y, Itoh M, Kawaji H, de Hoon M, et al. Redefinition of the Human Mast Cell Transcriptome by Deep-CAGE Sequencing. Blood (2014) 123(17):e58-67. doi: 10.1182/blood-2013-02-483792

21. McNeil BD, Pundir P, Meeker S, Han L, Undem BJ, Kulka M, et al. Identification of a Mast-Cell-Specific Receptor Crucial for Pseudo-Allergic Drug Reactions. Nature (2015) 519(7542):237-41. doi: 10.1038/nature14022

22. Subramanian H, Gupta K, Guo Q, Price R, Ali H. Mas-Related Gene X2 (MrgX2) Is a Novel G Protein-Coupled Receptor for the Antimicrobial Peptide LL-37 in Human Mast Cells: Resistance to Receptor Phosphorylation, Desensitization, and Internalization. J Biol Chem (2011) 286(52):44739-49. doi: 10.1074/jbc.M111.277152

23. Subramanian H, Gupta K, Lee D, Bayir AK, Ahn H, Ali H. beta-Defensins Activate Human Mast Cells Via Mas-related Gene X2. J Immunol (2013) 191 (1):345-52. doi: 10.4049/jimmunol.1300023

24. Zhang L, McNeil BD. Beta-Defensins Are Proinflammatory Pruritogens That Activate Mrgprs. J Allergy Clin Immunol (2019) 143(5):1960-2.e5. doi: 10.1016/j.jaci.2019.01.013

25. Lister PD, Wolter DJ, Hanson ND. Antibacterial-Resistant Pseudomonas Aeruginosa: Clinical Impact and Complex Regulation of Chromosomally Encoded Resistance Mechanisms. Clin Microbiol Rev (2009) 22(4):582-610. doi: 10.1128/CMR.00040-09

26. Serra R, Grande R, Butrico L, Rossi A, Settimio UF, Caroleo B, et al. Chronic Wound Infections: The Role of Pseudomonas Aeruginosa and Staphylococcus Aureus. Expert Rev Anti Infect Ther (2015) 13(5):605-13. doi: 10.1586/ 14787210.2015.1023291

27. Zimmermann C, Troeltzsch D, Gimenez-Rivera VA, Galli SJ, Metz M, Maurer $\mathrm{M}$, et al. Mast Cells Are Critical for Controlling the Bacterial Burden and the Healing of Infected Wounds. Proc Natl Acad Sci U S A (2019) 116(41):205004. doi: $10.1073 /$ pnas. 1908816116

28. Kokryakov VN, Harwig SS, Panyutich EA, Shevchenko AA, Aleshina GM, Shamova OV, et al. Protegrins: Leukocyte Antimicrobial Peptides That Combine Features of Corticostatic Defensins and Tachyplesins. FEBS Lett (1993) 327(2):231-6. doi: 10.1016/0014-5793(93)80175-T

29. Gupta K, Kotian A, Subramanian H, Daniell H, Ali H. Activation of Human Mast Cells by Retrocyclin and Protegrin Highlight Their Immunomodulatory and Antimicrobial Properties. Oncotarget (2015) 6(30):28573-87. doi: 10.18632/oncotarget.5611

30. Chowdhury MH, Ryan LK, Cherabuddi K, Freeman KB, Weaver DG, Pelletier JC, et al. Antifungal Potential of Host Defense Peptide Mimetics in a Mouse Model of Disseminated Candidiasis. J Fungi (Basel) (2018) 4(1):30. doi: 10.3390/jof4010030

31. Ryan LK, Freeman KB, Masso-Silva JA, Falkovsky K, Aloyouny A, Markowitz $\mathrm{K}$, et al. Activity of Potent and Selective Host Defense Peptide Mimetics in Mouse Models of Oral Candidiasis. Antimicrob Agents Chemother (2014) 58 (7):3820-7. doi: 10.1128/AAC.02649-13

32. Scott RW, Tew GN. Mimics of Host Defense Proteins; Strategies for Translation to Therapeutic Applications. Curr Top Med Chem (2017) 17 (5):576-89. doi: 10.2174/1568026616666160713130452

33. Ekkelenkamp MB, Canton R, Diez-Aguilar M, Tunney MM, Gilpin DF, Bernardini F, et al. Susceptibility of Pseudomonas Aeruginosa Recovered From Cystic Fibrosis Patients to Murepavadin and 13 Comparator Antibiotics. Antimicrob Agents Chemother (2020) 64(2):e01541-19. doi: 10.1128/AAC.01541-19

34. Tummler B. Emerging Therapies Against Infections With Pseudomonas Aeruginosa. F1000Res (2019) 8:1371. doi: 10.12688/f1000research.19509.1

35. Jabbour JF, Sharara SL, Kanj SS. Treatment of Multidrug-Resistant GramNegative Skin and Soft Tissue Infections. Curr Opin Infect Dis (2020) 33 (2):146-54. doi: 10.1097/QCO.0000000000000635

36. Cahill TJ3rd, Thomsen AR, Tarrasch JT, Plouffe B, Nguyen AH, Yang F, et al. Distinct Conformations of GPCR-beta-Arrestin Complexes Mediate Desensitization, Signaling, and Endocytosis. Proc Natl Acad Sci U S A (2017) 114(10):2562-7. doi: 10.1073/pnas.1701529114

37. Roy S, Ganguly A, Haque M, Ali H. Angiogenic Host Defense Peptide AG-30/ $5 \mathrm{C}$ and Bradykinin B2 Receptor Antagonist Icatibant Are G Protein Biased Agonists for MRGPRX2 in Mast Cells. J Immunol (2019) 202(4):1229-38. doi: 10.4049/jimmunol.1801227 
38. Alkanfari I, Freeman KB, Roy S, Jahan T, Scott RW, Ali H. Small-Molecule Host-Defense Peptide Mimetic Antibacterial and Antifungal Agents Activate Human and Mouse Mast Cells Via Mas-Related GPCRs. Cells (2019) 8(4):311. doi: 10.3390/cells8040311

39. Kirshenbaum AS, Akin C, Wu Y, Rottem M, Goff JP, Beaven MA, et al. Characterization of Novel Stem Cell Factor Responsive Human Mast Cell Lines LAD 1 and 2 Established From a Patient With Mast Cell Sarcoma/ Leukemia; Activation Following Aggregation of FcepsilonRI or FcgammaRI. Leuk Res (2003) 27(8):677-82. doi: 10.1016/S0145-2126(02)00343-0

40. Ali H, Richardson RM, Tomhave ED, DuBose RA, Haribabu B, Snyderman R. Regulation of Stably Transfected Platelet Activating Factor Receptor in RBL2H3 Cells. Role of Multiple G Proteins and Receptor Phosphorylation. J Biol Chem (1994) 269(40):24557-63. doi: 10.1016/S0021-9258(17)31428-X

41. Lansu K, Karpiak J, Liu J, Huang XP, McCorvy JD, Kroeze WK, et al. In Silico Design of Novel Probes for the Atypical Opioid Receptor MRGPRX2. Nat Chem Biol (2017) 13(5):529-36. doi: 10.1038/nchembio.2334

42. Chompunud Na Ayudhya C, Amponnawarat A, Roy S, Oskeritzian CA, Ali H. MRGPRX2 Activation by Rocuronium: Insights From Studies With Human Skin Mast Cells and Missense Variants. Cells (2021) 10(1):156. doi: 10.3390/ cells10010156

43. Chompunud Na Ayudhya C, Roy S, Alkanfari I, Ganguly A, Ali H. Identification of Gain and Loss of Function Missense Variants in MRGPRX2's Transmembrane and Intracellular Domains for Mast Cell Activation by Substance P. Int J Mol Sci (2019) 20(21):5247. doi: 10.3390/ ijms20215247

44. Alkanfari I, Gupta K, Jahan T, Ali H. Naturally Occurring Missense MRGPRX2 Variants Display Loss of Function Phenotype for Mast Cell Degranulation in Response to Substance P, Hemokinin-1, Human betaDefensin-3, and Icatibant. J Immunol (2018) 201(2):343-9. doi: 10.4049/ jimmunol.1701793

45. Martin-Loeches I, Dale GE, Torres A. Murepavadin: A New Antibiotic Class in the Pipeline. Expert Rev Anti Infect Ther (2018) 16(4):259-68. doi: 10.1080/ 14787210.2018.1441024

46. Srinivas N, Jetter P, Ueberbacher BJ, Werneburg M, Zerbe K, Steinmann J, et al. Peptidomimetic Antibiotics Target Outer-Membrane Biogenesis in Pseudomonas Aeruginosa. Science (2010) 327(5968):1010-3. doi: 10.1126/ science. 1182749

47. Wach A, Dembowsky K, Dale GE. Pharmacokinetics and Safety of Intravenous Murepavadin Infusion in Healthy Adult Subjects Administered Single and Multiple Ascending Doses. Antimicrob Agents Chemother (2018) 62(4):e02355-17. doi: 10.1128/AAC.02355-17
48. Diez-Aguilar M, Hernandez-Garcia M, Morosini MI, Fluit A, Tunney MM, Huertas N, et al. Murepavadin Antimicrobial Activity Against and Resistance Development in Cystic Fibrosis Pseudomonas Aeruginosa Isolates. J Antimicrob Chemother (2021) 76(4):984-92. doi: 10.1093/jac/dkaa529

49. Dwyer DF, Barrett NA, Austen KF. Immunological Genome Project C. Expression Profiling of Constitutive Mast Cells Reveals a Unique Identity Within the Immune System. Nat Immunol (2016) 17(7):878-87. doi: 10.1038/ ni.3445

50. Weller K, Foitzik K, Paus R, Syska W, Maurer M. Mast Cells Are Required for Normal Healing of Skin Wounds in Mice. FASEB J (2006) 20(13):2366-8. doi: 10.1096/fj.06-5837fje

51. McCaig LF, McDonald LC, Mandal S, Jernigan DB. Staphylococcus AureusAssociated Skin and Soft Tissue Infections in Ambulatory Care. Emerg Infect Dis (2006) 12(11):1715-23. doi: 10.3201/eid1211.060190

52. Moran GJ, Krishnadasan A, Gorwitz RJ, Fosheim GE, McDougal LK, Carey $\mathrm{RB}$, et al. Methicillin-Resistant S. Aureus Infections Among Patients in the Emergency Department. N Engl J Med (2006) 355(7):666-74. doi: 10.1056/ NEJMoa055356

53. DeWire SM, Ahn S, Lefkowitz RJ, Shenoy SK. Beta-Arrestins and Cell Signaling. Annu Rev Physiol (2007) 69:483-510. doi: 10.1146/ annurev.physiol.69.022405.154749

54. Lefkowitz RJ. Arrestins Come of Age: A Personal Historical Perspective. Prog Mol Biol Trans Sci (2013) 118:3-18. doi: 10.1016/B978-0-12-394440-5.00001-2

55. Shenoy SK, Lefkowitz RJ. Beta-Arrestin-Mediated Receptor Trafficking and Signal Transduction. Trends Pharmacol Sci (2011) 32(9):521-33. doi: 10.1016/ j.tips.2011.05.002

56. Ranjan R, Pandey S, Shukla AK. Biased Opioid Receptor Ligands: Gain Without Pain. Trends Endocrinol Metab (2017) 28(4):247-9. doi: 10.1016/ j.tem.2017.01.001

Conflict of Interest: The authors declare that the research was conducted in the absence of any commercial or financial relationships that could be construed as a potential conflict of interest.

Copyright (C) 2021 Amponnawarat, Chompunud Na Ayudhya and Ali. This is an open-access article distributed under the terms of the Creative Commons Attribution License (CC BY). The use, distribution or reproduction in other forums is permitted, provided the original author(s) and the copyright owner(s) are credited and that the original publication in this journal is cited, in accordance with accepted academic practice. No use, distribution or reproduction is permitted which does not comply with these terms. 